\title{
Tumor neuroendocrino en recto simulando una neoplasia benigna: reporte de caso y revisión de la literatura
}

\author{
Neuroendocrine tumor in the rectum simulating a benign neoplasm: \\ case report and literature review
}

\author{
Katherine Redondo de Oro' ${ }^{1}$, Cesar Redondo-Bermúdez¹, Luis David Mendoza-Durán², \\ Valentina Marrugo-Padilla², Marian Selena Otero-Urda ${ }^{3}$
}

1 Médico, especialista en Patología, Departamento de Patología; Grupo de Investigación Histopatología, Universidad de Cartagena, Cartagena, Colombia.

2 Estudiante de Medicina, Universidad de Cartagena; Grupo de Investigación, Centro de Investigaciones Biomédicas, Cartagena, Colombia.

3 Médico, Universidad de Cartagena, Cartagena, Colombia.

\section{Resumen}

Los tumores neuroendocrinos se definen como un grupo heterogéneo de neoplasias de origen epitelial, provenientes de células enterocromafines diseminadas por todo el organismo, y representan alrededor del 1 al $4 \%$ de todas las neoplasias. Su mayor distribución se encuentra en el tracto gastrointestinal, donde se localiza el 75 \% de los tumores neuroendocrinos, siendo los ubicados en el recto, el $27 \%$ de todos los que afectan el tracto gastrointestinal. A propósito de esta revisión de tema, presentamos el caso de un paciente de 71 años de edad, que consultó por sangrado rectal rojo rutilante, sin otra sintomatología asociada, y se le diagnosticó un tumor neuroendocrino grado 1, que se comportaba como una lesión benigna del recto.

Palabras claves: neoplasias del recto; carcinoma neuroendocrino; células enterocromafines; diagnóstico; endoscopía del sistema digestivo.

\begin{abstract}
Neuroendocrine tumors are defined as a heterogeneous group of neoplasms of epithelial origin from enterochromaffin cells disseminated throughout the body, and represent about $1 \%$ to $4 \%$ of all neoplasms. Its largest distribution is found in the gastrointestinal tract, where $75 \%$ of neuroendocrine tumors are located, being $27 \%$ of those in the rectum. We present the case of a 71-year-old patient who consulted for bright red blood per rectum, with no other associated symptoms, and was diagnosed with a grade 1 neuroendocrine tumor, which behaved as a benign lesion of the rectum.
\end{abstract}

Keywords: rectal neoplasms; carcinoma, neuroendocrine; enterochromaffin cell; diagnosis; endoscopy, digestive system.

Fecha de recibido: 02/11/2020 - Fecha de aceptación: 19/02/2021 - Fecha de publicación en línea: 10/06/2021

Correspondencia: Katherine Redondo de Oro, Código postal: 130001, Cartagena de Indias, Colombia. Teléfono: 3003045313. Dirección electrónica: kredondod@unicartagena.edu.co

Citar como: Redondo de Oro K, Redondo-Bermúdez C, Mendoza-Durán LD, Marrugo-Padilla V, Otero-Urda MS . Tumor neuroendocrino en recto simulando una neoplasia benigna: reporte caso y revisión de la literatura. Rev Colomb Cir. 2021;36:696-702. https://doi.org/10.30944/20117582.813

Este es un artículo de acceso abierto bajo una Licencia Creative Commons - BY-NC-ND https://creativecommons.org/licenses/by-ncnd/4.0/deed.es 


\section{Definición}

Los tumores neuroendocrinos (TNE) son neoplasias infrecuentes, de origen epitelial. Proceden de células enterocromafines, también llamadas neuroendocrinas, diseminadas por todo el organismo ${ }^{1}$.

\section{Epidemiología}

La incidencia anual de los TNE es de 1,04 por cada 100.000 personas. Ocasionan el $1-4 \%$ de todas las neoplasias ${ }^{2}$. No obstante, los TNE de colon y recto presentan una alta prevalencia, siendo considerados como la segunda neoplasia más prevalente en esa ubicación, después del adenocarcinoma colorrectal $^{3}$.

En lo referente a su localización, se presentan con mayor frecuencia en tracto gastrointestinal, páncreas y pulmón ${ }^{4}$. Esto se debe a que existe una mayor población de células enterocromafines en estos sitios. Es por ello que, el $75 \%$ de los TNE tienen su ubicación en el tracto gastrointestinal ${ }^{5}, \mathrm{y}$ de ellos, el $27 \%$ se encuentran en el recto, siendo este último la tercera ubicación más frecuente entre los órganos del tracto gastrointestinal, después del apéndice cecal y el íleon ${ }^{1,2}$.

La presentación de TNE localizados en recto (TNE-R) es muy infrecuente, llegando a causar el $2 \%$ de todos los tumores rectales ${ }^{1}$. Sin embargo, su incidencia ha ido en aumento en las tres últimas décadas, alcanzando el $18 \%$ de todos los TNE y el $27 \%$ de los TNE gastroenteropancreáticos (TNEGEP) ${ }^{6,7}$. Algunos autores incluso afirman que los TNE-R superan en frecuencia a los TNE en íleon ${ }^{8}$.

La mayoría de los portadores de estas neoplasias se encuentran entre la sexta y la séptima década de la vida. La edad promedio al momento del diagnóstico es de 56 años.

Existe poca diferencia en la distribución entre hombres y mujeres, pues afecta ligeramente más a hombres que a mujeres, con una proporción de 1,1 $: 1$. Son más frecuentes en varones de raza negra, aunque parece existir cierta predisposición por los asiáticos $6,8,9$.

No se han establecido factores de riesgo para los TNE y esto se debe a su baja incidencia, pero se han encontrado asociaciones entre niveles altos de colesterol, síndrome metabólico y antecedentes familiares de cáncer ${ }^{8}$.

\section{Sintomatología}

Los TNE pueden tener características funcionales o carecer de ellas, esto es debido a que provienen de células productoras de hormonas ${ }^{10-12}$. En su mayoría los tumores no son funcionales. Sin embargo, cuando lo son, tienden a no generar síntomas en etapas iniciales, porque el hígado metaboliza las hormonas producidas por el tumor, lo que se conoce como efecto de primer paso, y ocurre principalmente en tumores desarrollados en intestino delgado o colon ${ }^{10,13}$.

En etapas avanzadas, una vez los TNE hacen metástasis a hígado o la producción de hormonas supera su metabolismo, los síntomas hormonales se manifiestan en los pacientes y el tumor se nombra de acuerdo a la hormona que el mismo produce, por ejemplo, síndrome carcinoide, insulinoma, o gastrinoma, entre otros ${ }^{10,12,14}$.

\section{Presentación clínica}

Los TNE son diagnosticados de manera incidental debido a su crecimiento lento y los síntomas vagos que generan. Los TNE-R suelen presentarse clínicamente como cambios en los hábitos intestinales, sangrado oculto en las heces, dolor abdominal, cuadros obstructivos, pérdida de peso o rectorragia $^{8}$. No obstante, casi el $50 \%$ de ellos tienen una presentación asintomática, ya que en la mayoría de los casos son no funcionales y, por lo tanto, carecen de manifestaciones clínicas concordantes con las hormonas que producen, tal como la serotonina.

Con mayor frecuencia, los TNE-R se detectan de forma incidental durante la realización de colonoscopias indicadas por otras razones ${ }^{6,8}$. La biopsia es la prueba estándar para el diagnóstico de los TNE y la más utilizada durante la realización de endoscopias en lesiones sospechosas de colon, recto, estómago y duodeno, además de que permite estudiar las características del tumor y su respectiva gradación ${ }^{5,10}$. 


\section{Histopatología}

Las características macroscópicas de los TNE pueden variar según el tamaño del tumor y el grado de diferenciación. Generalmente son lesiones sólidas, localizadas en la mucosa profunda o submucosa ${ }^{3,7}$, pequeñas (diámetro menor de un $\mathrm{cm}$ ), blanquecino-amarillentas o grisáceas, con aspecto polipoide o nodular, y con bordes bien delimitados, con invasión de tejido adyacente tipo empuje. Puede tener áreas hemorrágicas o ulceraciones y, en raras ocasiones, lesiones quísticas causadas por degeneración central ${ }^{8}$.

A nivel microscópico, los TNE bien diferenciados se caracterizan por presentar proliferación de células redondas u ovoides, con núcleos de tamaño aumentado y citoplasma amplio, anfofílico o ligeramente eosinofílico ${ }^{7}$. Además, pueden tener apariencia plasmocitoide, con núcleos excéntricos y una cantidad moderada de citoplasma.

Estas células pueden disponerse en diferentes patrones, como anidados, solidos, trabeculares, pseudoglandulares y giriformes. Por otro lado, el estroma se muestra fibroso o, en algunos casos, con hialinización ${ }^{7}$. En el caso de TNE bien diferenciados, se observa proliferación de células tumorales en forma de hoja, con núcleos irregulares, características mitóticas elevadas y menor número de gránulos de secreción citoplasmáticos ${ }^{13}$.

\section{Clasificación}

Los TNE-GEP son clasificados histológicamente según los valores del conteo mitótico y el índice de proliferación Ki-67 establecidos por la OMS en su 5aㅡ decisión en el $2019^{11}$, independientemente del origen, el tamaño y la extensión anatómica del tumor ${ }^{15}$. Ambos aspectos deben ser evaluados en los denominados puntos calientes del tumor, que representan las regiones con mayor actividad celular, según se observa en la exploración microscópica de ampliación intermedia ${ }^{7}$.

Los TNE-GEP se clasifican de la siguiente manera ${ }^{3,11,15}$ :

- TNE bien diferenciados:

- Grado 1 con un conteo mitótico menor de 2/10 campos microscópicos de alta poten- cia (CAP) y un índice Ki-67 menor de $3 \%$.

- Grado 2 con conteo mitótico de 2-20/10 CAP y un índice Ki-67 de 3-20 \%.

- Grado 3 con conteo mitótico mayor de 20/10 CAP y un índice Ki-67 mayor de 20 \%.

- CNE o TNE mal diferenciado:

- Grado 3 con conteo mitótico mayor de 20/10 CAP y un índice Ki-67 mayor de $20 \%$, tiene un componente más agresivo y presenta una variedad de células pequeñas y células grandes, similar al carcinoma de pulmón.

- Neoplasia mixta neuroendocrina - no neuroendocrina

Cabe resaltar que en la nueva clasificación de los TNE-GEP, el grado 3 y los carcinomas neuroendocrinos (CNE) son difíciles de diferenciar morfológicamente y no existe un valor de Ki-67 para distinguir estas dos neoplasias. Pero existen características moleculares, como la pérdida de expresión de DAXX o ATRX y unos valores elevados de cromogranina-A en plasma, que son específicos de los TNE grado 3, mientras que en los CNE mal diferenciados se identifica comúnmente la pérdida de $\mathrm{Rb}$ o de la expresión anormal de p53. Además, el estudio inmunohistoquímico adicional puede ayudar en la diferenciación de estas dos entidades ${ }^{15}$.

En la nueva clasificación se establece también la existencia de un grupo de tumores considerados mixtos, llamados neoplasia mixta neuroendocrina no neuroendocrina, que anteriormente eran nombrados carcinomas adenoneuroendocrino mixtos. Ahora son considerados dentro de los TNE grado 3 , independiente del conteo mitótico y el índice de proliferación Ki-67 ${ }^{16}$. Estos tumores contienen un componente neuroendocrino y otro no endocrino, considerándose la coexistencia entre un TNE y un adenocarcinoma ${ }^{3}$. El componente no neuroendocrino, con mayor frecuencia, es el carcinoma escamoso ${ }^{8}$.

Los marcadores de diferenciación neuroendocrina utilizados para teñir las muestras y obtener 
los gránulos de neurosecreción por inmunohistoquímica son la cromogranina A y la sinaptofisina, como los más específicos, encontrándose una expresión más difusa e intensa en esta última ${ }^{11}$. También el CD56, CD57, CDX2 y la enolasa neuronoespecífica, entre otras ${ }^{3,7}$.

\section{Diagnóstico}

El diagnóstico definitivo se realiza a través de la biopsia, la cual se practica generalmente (40\%) durante una colonoscopia, en algunos casos por razones diferentes a la búsqueda de lesiones tumorales ${ }^{8}$. Al momento del diagnóstico, aproximadamente el $80 \%$ son tumores menores de 1 $\mathrm{cm}$, un $15 \%$ están entre 1 y $2 \mathrm{~cm}$ y, los mayores de $2 \mathrm{~cm}$ representan el $5 \%{ }^{1}$. Además, la mayoría son identificados en el recto medio $(5-10 \mathrm{~cm}$ del borde anal) ${ }^{6}$.

El uso de imágenes para el diagnóstico de TNE en recto no está indicado, debido a que, en su mayoría, pueden ser tratados endoscópicamente ${ }^{1}$. Sin embargo, en los TNE con tamaño de más de 1 cm de diámetro o con reporte de características de alto riesgo, se debe considerar el uso de resonancia magnética nuclear (RMN) de pelvis o ecografía endorrectal, con el fin de evaluar invasión a tejidos subyacentes y el estado de los ganglios linfáticos, y en tumores mayores de $2 \mathrm{~cm}$, se recomienda el uso de tomografía computarizada (TC) o RMN de abdomen y pelvis, para buscar metástasis ${ }^{6,8}$.

Las metástasis son raras y en su mayoría no funcionales. Afectan más frecuentemente el hígado y los ganglios linfáticos ${ }^{4}$, pero también se han reportado en hueso e incluso pulmón ${ }^{4,17}$.

El avance de las técnicas de imagen y la identificación de los receptores de somatostatina (SSTR), los cuales se encuentran sobreexpresados en TNE, han permitido una mejor identificación de la enfermedad con el uso de análogos de somatostatina radiomarcados ${ }^{1}$. Esto, que ha sido denominado SSTR-PET, debe combinar el contraste intravenoso con TC o RMN. La PET con TC ofrece una mejor visualización de enfermedad mesentérica, ósea y pulmonar, mientras que la PET con RMN visualiza mejor las metástasis hepáticas. Sin embargo, en el caso de tumores de bajo riesgo, como TNE en recto menor de $1 \mathrm{~cm}$ de diámetro, se considera esta opción para la estadificación ${ }^{1,8}$.

\section{Tratamiento}

El tratamiento de los TNE va a depender del tamaño del tumor. Aproximadamente, el $80 \%$ de los TNE-R pueden ser tratados con endoscopia ${ }^{3}$. Para los tumores en recto de menos de $1 \mathrm{~cm}$, el tratamiento es la resección local, ya sea a través de un procedimiento endoscópico o transanal. En cuanto al procedimiento endoscópico, este incluye polipectomía tradicional, resección de mucosa, ligadura con banda y disección endoscópica submucosa ${ }^{3,18}$.

En el caso de los TNE de tamaño entre 1-2 cm, se debe realizar una ecografía endorrectal y/o una RMN pélvica, para determinar si presenta invasión de la muscular propia o metástasis ganglionar regional. Una vez descartados estos, los tumores pueden ser removidos con los procedimientos anteriormente mencionados, favoreciendo las técnicas que faciliten la resección en bloque con bordes negativos o los abordajes quirúrgicos transanales para las lesiones más grandes. En caso de invasión de la muscular propia o ganglios linfáticos positivos, se debe considerar la excisión mesorrectal radical con resección anterior baja o abdominoperineal $^{1,18}$.

Por último, los tumores mayores de $2 \mathrm{~cm}$ en su mayoría son considerados de alto grado por su mala diferenciación, invasión de la muscular propia e invasión linfática. Por estas características se debe realizar una resección anterior baja o abdominoperineal ${ }^{8}$. El tratamiento con quimioterapia podría estar indicado en TNE mal diferenciados ${ }^{6}$.

\section{Pronóstico}

El pronóstico de los TNE-R depende de aspectos como el tamaño, el estado de los márgenes, el grado del mismo según la clasificación de la OMS, la diferenciación histológica y el sitio de origen ${ }^{3,7}$. Al momento del diagnóstico, entre el $75 \%$ y el $85 \%$ de las lesiones se encuentran localizadas, con un rango de sobrevida a los 5 años del $75-93 \%^{1,3}$. En el caso de los TNE bien o moderadamente diferenciados, el pronóstico varía en función a su grado: 
92-100 \% para grado $1,80-88 \%$ para grado 2 , $51-59 \%$ para grado 3 y 0-15\% para carcinoma neuroendocrino ${ }^{6}$.

Si el tamaño del tumor es menor a $1 \mathrm{~cm}$, tienen una baja probabilidad de afectación de la muscular propia, vasos sanguíneos o linfáticos, tienen índices mitóticos menores de 2/10 CAP y muestran metástasis en menos del $2 \%$ de los pacientes ${ }^{1}$. Sin embargo, se han reportado casos de tumores menores de $1 \mathrm{~cm}$ con metástasis a hígado ${ }^{4}$. En el caso de tumores con tamaño mayor a $2 \mathrm{~cm}$, la probabilidad de presentar enfermedad metastásica es de un 60 a $80 \%$, mientras que en tumores entre 1 y $1,9 \mathrm{~cm}$, su riesgo de metástasis es intermedio $(15 \%)^{8}$. Se ha propuesto un límite de 1 a 1,4 cm para el riesgo de metástasis, invasión linfática, vascular e índice mitótico menor de 2/10 CAP.

La invasión de la muscular propia aumenta el riesgo de metástasis en estos tumores ${ }^{1}$. La supervivencia a cinco años varía de 15 a 32\% si hay metástasis a distancia ${ }^{13}$. La presencia de ulceración, hiperemia o depresión central se asocia a invasión de tejido adyacente y linfático, empeorando el pronóstico ${ }^{6}$. Por último, se recomienda la combinación del estadio TNM con el grado tumoral, que son los elementos pronósticos más relevantes ${ }^{19}$.

\section{Presentación de caso}

Como complemento de esta revisión de tema, presentamos el caso de un hombre de 71 años, con antecedentes de infarto agudo de miocardio, hipertensión arterial, dislipidemia, pólipos en colon y patología hemorroidal, que asiste a consulta por un episodio de sangrado rectal, rojo rutilante, sin otra sintomatología asociada. A la colonoscopia se describe a nivel del recto una lesión subepitelial, bien delimitada, regular, de aproximadamente 0,3 $\mathrm{cm}$, que sugiere un lipoma u otro tumor benigno del tracto gastrointestinal.

En la biopsia se reporta una lesión tumoral constituida por proliferación de células redondas, de tamaño intermedio, con núcleos hipercromáticos, que se disponen en pequeños nidos con patrón infiltrativo (Figura 1), sugiriendo como diagnóstico, por el patrón morfológico, un tumor de origen neuroendocrino. El diagnóstico fue confirmado con estudios de inmunohistoquímica, ya que las células tumorales expresaron CKAE1/AE3, Sinaptofisina y CD56, fueron negativas con CDX2 y Enolasa. El índice de proliferación celular evaluado con el K167 fue menor al $1 \%$, con lo que se hace el diagnóstico de tumor neuroendocrino bien diferenciado grado 1.

Así mismo, se realizó una RNM para evaluar extensión del tumor, donde se observa marcado engrosamiento de la pared rectal, sin tumor residual, producto del procedimiento quirúrgico y la reacción inflamatoria reparativa (Figura 2). La colonoscopia de control solo evidenció cicatriz sin cambios displásicos (Figura 3).
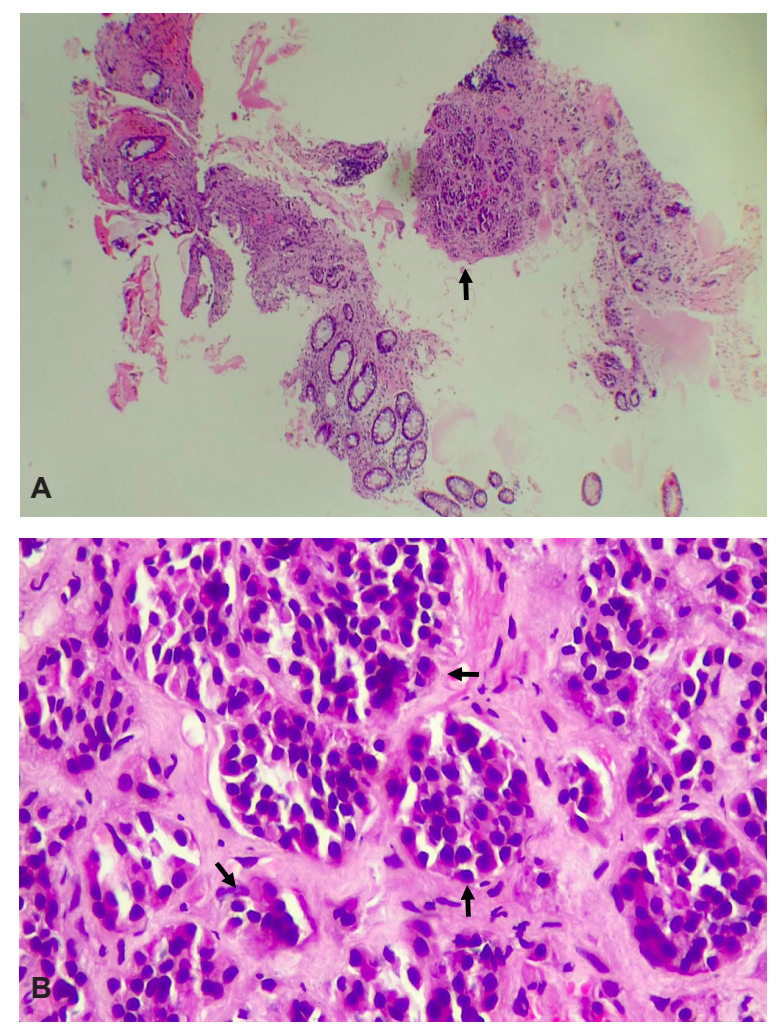

Figura 1. A. 10x. Cortes histológicos donde se aprecia la mucosa colónica con una lesión tumoral constituida por pequeños acinos bien definidos (flecha), escasamente representados en biopsia de colon. B. 40X. Los acinos están constituidos por células de tamaño intermedio a pequeños, con núcleos redondos a ovales, hipercromáticos, con actividad mitótica mínima a ausente en el material evaluado (flechas). 


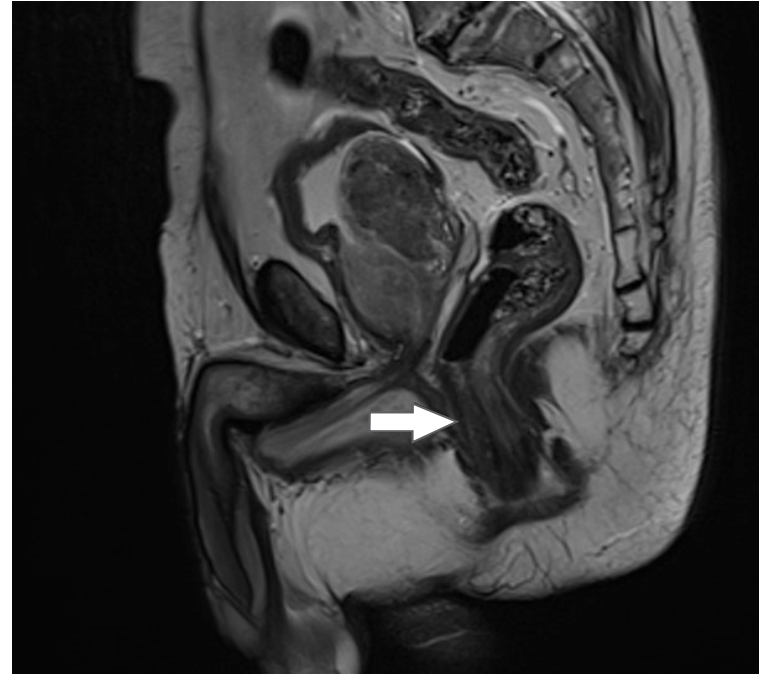

Figura 2. Se muestra corte sagital de resonancia magnética contrastada del paciente en la región abdominopélvica donde se observa engrosamiento de la pared del recto (flecha), sin lesión residual.

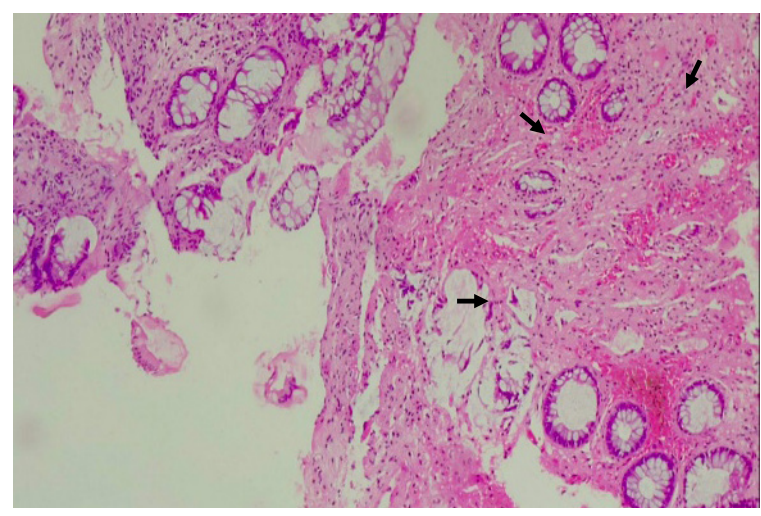

Figura 3. 40X. Los cortes histológicos revelan mucosa colorrectal con marcada disminución del componente glandular, rodeada por una lámina propia con marcada fibrosis (flechas), sin evidencia de lesión residual.

El paciente posteriormente ha evolucionado de manera satisfactoria, continua asintomático, de buen estado general y con controles de colonoscopia y RMN dentro de límites normales. No requirió tratamiento adicional.

\section{Conclusiones}

Los TNE son neoplasias infrecuentes en el organismo. A pesar de ello, los TNE-R han venido aumentando su incidencia, lo que se ha hecho notable gracias al mayor uso de endoscopias que los detectan de forma incidental, alcanzando a representar el $27 \%$ de todos los tumores del tracto gastrointestinal. Estos suelen ser asintomáticos, aunque algunos pueden tener ciertas manifestaciones clínicas, como cambios en el hábito intestinal, pérdida de peso y rectorragia, tal fue el caso de nuestro paciente.

Las características propias del tumor, como el tamaño, el estado de los márgenes, el grado según la clasificación de la OMS y otros factores asociados, determinan el pronóstico de vida y el riesgo de metástasis en los pacientes con TNE en recto. Los tumores con tamaño menor de $1 \mathrm{~cm}$ se asocian con una supervivencia mayor del $92 \%$ a 5 años.

El desarrollo de nuevas técnicas de imágenes diagnósticas y el uso de endoscopia como método de tamizaje para neoplasias colorrectales contribuirán en la detección más temprana de estas neoplasias, lo que a su vez permite ofrecer mejores posibilidades de tratamiento a los pacientes que desarrollen TNE-R.

\section{Cumplimiento de normas éticas}

Consentimiento informado: Este estudio es una revisión de la literatura. Se adjunto un caso clínico, pero no se incluyeron imágenes que permitan la identificación del paciente, por lo que no hay necesidad de un consentimiento informado.

Declaración de conflicto de intereses: Los autores declaran que no tienen conflictos de intereses.

Fuentes de financiación: Recursos propios de los autores. La realización de este artículo no fue financiada por ninguna institución u organización.

\section{Contribuciones de los autores}

- Concepción y diseño del estudio: Katherine Redondo de Oro, Cesar Redondo-Bermúdez.

- Adquisición de datos: Katherine Redondo de Oro, Marian Otero-Urda, Luis Mendoza-Durán.

- Análisis e interpretación de datos: Katherine Redondo de Oro, Marian Otero, Luis Mendoza-Durán, Valentina Marrugo-Padilla.

- Redacción del manuscrito: Katherine Redondo de Oro, Marian Otero, Luis Mendoza-Durán, Valentina MarrugoPadilla.

- Revisión crítica: Katherine Redondo de Oro, Cesar Redondo-Bermúdez. 


\section{Referencias}

1. Bertani E, Ravizza D, Milione M, Massironi S, Grana CM, Zerini D, et al. Neuroendocrine neoplasms of rectum: A management update. Cancer Treat Rev. 2018;66:45-55. https://doi.org/10.1016/j.ctrv.2018.04.003

2. Pérez-Sánchez E, Hernández-Barroso M, Hernández-Hernández G, Gambra-Michel L, Barrera-Gómez M. Carcinoma neuroendocrino rectal: Una neoplasia infrecuente. Rev Colomb Cancerol. 2018;22:126-9.

3. Bosolino A, Ratto R. Tumores neuroendocrinos de colon y recto. Acta Gastroenterol Latinoam. 2018;48:328-38.

4. Nagata K, Tajiri K, Shimada S, Ando T, Hosokawa A, Matsui K, et al. Rectal neuroendocrine tumor G1 with a solitary hepatic metastatic lesion. Intern Med. 2017;56:289-93.

https://doi.org/10.2169/internalmedicine.56.7523

5. Nutu OA, Brandáriz L, Pérez-Carreras M, García-Borda J, Perea J. Tumor neuroendocrino de recto de comportamiento excepcional. Gastroenterol Hepatol. 2017;40:351-2.

https://doi.org/10.1016/j.gastrohep.2016.03.009

6. De Andrés-Asenjo B, Ortíz de Solórzano-Aurusa FJ, Borrego-Pintado H, Blanco-Antona F, Romero-de DIego A, Beltrán de Heredia-Rentería J. Tumor neuroendocrino ano-rectal: desde un pólipo con buen pronóstico hasta un carcinoma letal. Cir Cir. 2018;86:515-21. https://doi.org/10.24875/CIRU.18000302

7. Patel N, Barbieri A, Gibson J. Neuroendocrine tumors of the gastrointestinal tract and pancreas. Surg Pathol Clin. 2019;12:1021-44. https://doi.org/10.1016/j.path.2019.08.007

8. Hrabe J. Neuroendocrine tumors of the appendix, colon, and rectum. Surg Oncol Clin N Am. 2020;29:267-79. https://doi.org/10.1016/j.soc.2019.11.010

9. Takita M, Sakai E, Nakao T, Kimoto Y, Ishii R, Konishi $\mathrm{T}$, et al. Clinical outcomes of patients with small rectal neuroendocrine tumors treated using endoscopic submucosal resection with a ligation device. Digestion. 2018;99:72-8. https://doi.org/10.1159/000494416
10. Bonds M, Rocha FG. Neuroendocrine tumors of the pancreatobiliary and gastrointestinal tracts. Surg Clin North Am. 2020;100:635-48.

https://doi.org/10.1016/j.suc.2020.02.010

11. Bellizzi AM. Pathologic considerations in gastroenteropancreatic neuroendocrine tumors. Surg Oncol Clin N Am. 2020;29:185-208.

https://doi.org/10.1016/j.soc.2019.11.003

12. Flórez NF, Pérez JC, Turizo A, Cuesta DP. Caracterización de pacientes con tumores neuroendocrinos en un hospital de referencia de alta complejidad. Rev Colomb Cirugía. 2020;35:639-46.

https://doi.org/10.30944/20117582.786

13. Oronsky B, Ma PC, Morgensztern D, Carter CA. Nothing but NET: A review of neuroendocrine tumors and carcinomas. Neoplasia. 2017;19:991-1002.

https://doi.org/10.1016/j.neo.2017.09.002

14. Rubin de Celis-Ferrari AC, Glasberg J, Riechelmann RP. Carcinoid syndrome: Update on the pathophysiology and treatment. Clinics. 2018;73(Suppl 1):e490s. https://doi.org/10.6061/clinics/2018/e490s

15. Kim JY, Hong SM, Ro JY. Recent updates on grading and classification of neuroendocrine tumors. Ann Diagn Pathol. 2017;29:11-6. https://doi.org/10.1016/j.anndiagpath.2017.04.005

16. Gonzalez RS. Diagnosis and management of gastrointestinal neuroendocrine neoplasms. Surg Pathol Clin. 2020;13:377-97. https://doi.org/10.1016/j.path.2020.04.002

17. Yuan H, Yang Y, Wang W, Cheng Y. A case report of neuroendocrine tumor (G3) at lower rectum with liver metastasis. Medicine. 2018;97:e12423. https://doi.org/10.1097/MD.0000000000012423

18. Ramage JK, De Herder WW, Delle Fave G, Ferolla P, Ferone $\mathrm{D}$, Ito $\mathrm{T}$, et al. ENETS consensus guidelines update for colorectal neuroendocrine neoplasms. Neuroendocrinology. 2016;103:139-43. https://doi.org/10.1159/000443166

19. Bannura G, Barrera A, Melo C, Illanes F, Gallardo C. Tumores neuroendocrinos primarios de colon y recto. Rev Chil cirugía. 2018;70:53-8. 